Special issue of the 2nd International Conference on Computational and Experimental Science and Engineering (ICCESEN 2015)

\title{
Effect of the Level Density Parameter Ratio on the Cross Sections of Fission of Uranium Isotopes
}

\author{
N. KARPUZ* \\ Amasya University, Sabuncuoğlu Şerefeddin Health Services Vocational School, Amasya, Turkey
}

Nuclear level density is a fission characteristics for each core. Level density parameter ratio $a_{f} / a_{n}$ plays an important role in the statistical calculations of the fission, investigation of heavy ion collisions and calculation of the reaction cross sections. In this study, the effects of the different level density ratio on the fission reaction cross section for uranium isotopes were investigated. Calculations were carried out using the Monte Carlo simulation codes ALICE/ASH and TALYS 1.6. The calculated results were compared with the experimental reaction cross sections from literature.

DOI: 10.12693/APhysPolA.130.306

PACS/topics: 25.85-w, 25.85.Ge

\section{Introduction}

In nuclear fission the nucleus evolves from a single ground state shape into two separate fission fragments. During the change in shape and configuration, the potential energy of the system initially increases, up to the fission barrier height, and then decreases. Calculations of fission barriers involve the determination of the total nuclear potential energy for different nuclear shapes. The fission barrier height could be obtained from the energy relative to the ground state of the most favorable saddle point to be traversed when the shape evolves from a single shape to separated fragments [1]. The nuclear level density which is a characteristic property of every nucleus, is defined as the number of levels per unit energy at a certain excitation energy. Level density studies have very important applications in nuclear astrophysics, production cross sections of radioactive isotopes, nuclear fission and nuclear medicine.

The most important step for a reliable theoretical analysis of cross sections, spectra, angular distributions, and other nuclear reaction observables, is to get a correct level density together with the optical model potential [2]. Level density parameter ratio, $a_{f} / a_{n}$ where $a_{f}$ is the level density parameter at the fission saddle point and $a_{n}$ is the level density parameter after neutron evaporation, plays an important role in fission calculations. The choice of appropriate $a_{f} / a_{n}$ values stands as a problem in the calculations [3].

Radiation therapy is a form of cancer treatment that uses radiation to destroy malignant cells. For this purpose, ${ }^{137} \mathrm{Cs}$ is used because of its appropriate half-live value. It is produced in the fission the ${ }^{235} \mathrm{U}$ core. Therefore $(\mathrm{n}, \mathrm{f})$ reaction cross section for ${ }^{235} \mathrm{U}$ has been calculated in [4]. At the same time, some other isotopes

*e-mail: nurdankarpuz@gmail.com such as iodine are also vital, especially in medical science, and thus the production of these isotopes becomes important. ${ }^{125} \mathrm{Te}(\mathrm{p}, \mathrm{xn})$ reaction, in which iodine can be produced, was calculated for the $5.5-100.5 \mathrm{MeV}$ energy range using TALYS code. The calculated ${ }^{125} \mathrm{Te}(\mathrm{p}, \mathrm{xn})$ cross sections have been compared with results obtained by ALICE/ASH code [5]. Aside from the medical studies, TALYS code was also used in photonuclear applications. Zirconium is a very strong metal and it is the best construction material for elements of nuclear reactors, like cladding of fuel elements, due to its low neutron cross-section. The zirconium is also a potential target for the production of medical isotopes. For this purpose, the cross sections of $(\gamma, \mathrm{n})$ reaction in ${ }^{\text {nat }} \mathrm{Zr},{ }^{90} \mathrm{Zr},{ }^{91} \mathrm{Zr}$, ${ }^{92} \mathrm{Zr},{ }^{94} \mathrm{Zr}$ and ${ }^{96} \mathrm{Zr}$ targets have been calculated using the TALYS code [6]. The photonuclear reaction cross sections, especially the photoneutron reaction cross sections, are the fundamental properties of nuclear systems. Therefore, theoretical evaluation of photoneutron reaction cross sections for $27 \leq \mathrm{A} \leq 238$ targets $\left({ }^{27} \mathrm{Al},{ }^{96} \mathrm{Mo}\right.$, ${ }^{181} \mathrm{Ta},{ }^{238} \mathrm{U}$ ) have been investigated in the incident photon energy range of 7-35 MeV [7]. The cross section for $(\gamma, \mathrm{n})$ reaction is important for investigation of nuclear structure, especially in low-energy giant dipole resonance. The total cross sections of ${ }^{12} \mathrm{C}(\gamma, \mathrm{n})$ and ${ }^{12} \mathrm{C}(\gamma, \mathrm{p})$ reactions were calculated using TALYS nuclear code. The effect of the gamma strength function on the cross section exchange data has determined the most compatible model type [8].

In this study, the cross section of the proton-induced fission of uranium isotopes was calculated to show the dependence on the choice of the ratio of the level density parameters, $a_{f} / a_{n}$, by using ALICE/ASH [9] and TALYS $1.6[10]$ nuclear reaction simulation codes. The calculations made by using ALICE/ASH and TALYS 1.6 codes are compared with the existing experimental data, EXFOR [11]. The obtained results have been compared with the experimental results of Boyce et al. [12, 13], Ohtsuki et al. [14] and Smirnov et al. [15]. 


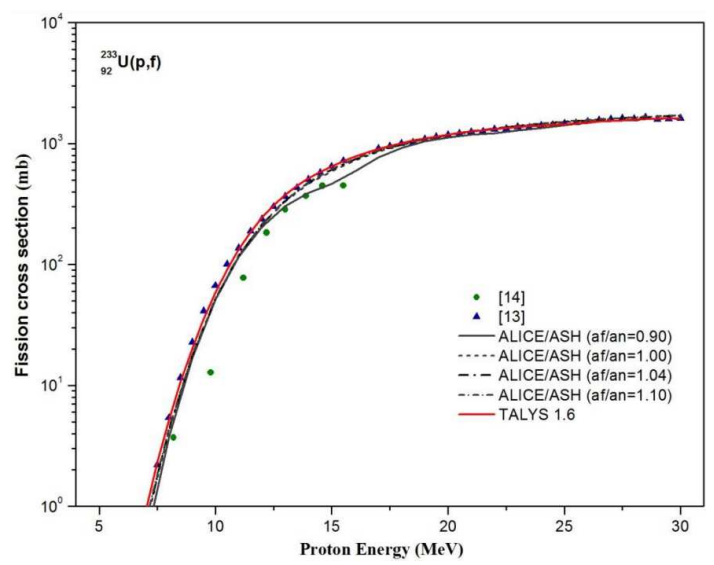

Fig. 1. Effect of the level density parameter ratio on fission cross sections for proton induced fission in ${ }^{233} \mathrm{U}$. The experimental data are taken from EXFOR.

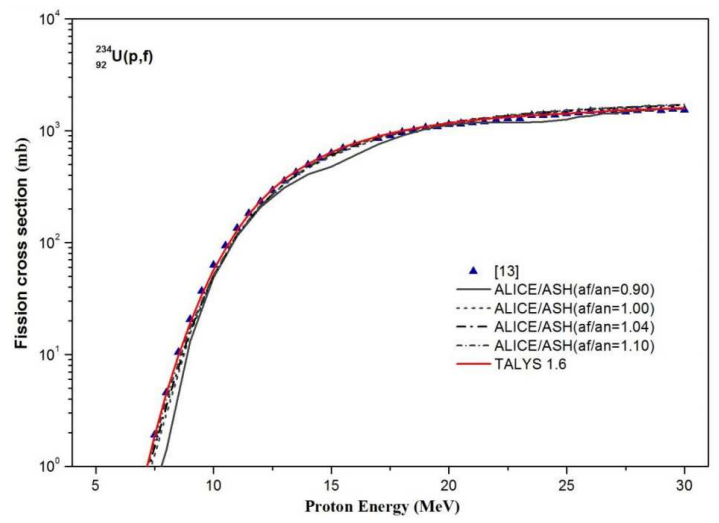

Fig. 2. As in Fig. 1, for ${ }^{234} \mathrm{U}$.

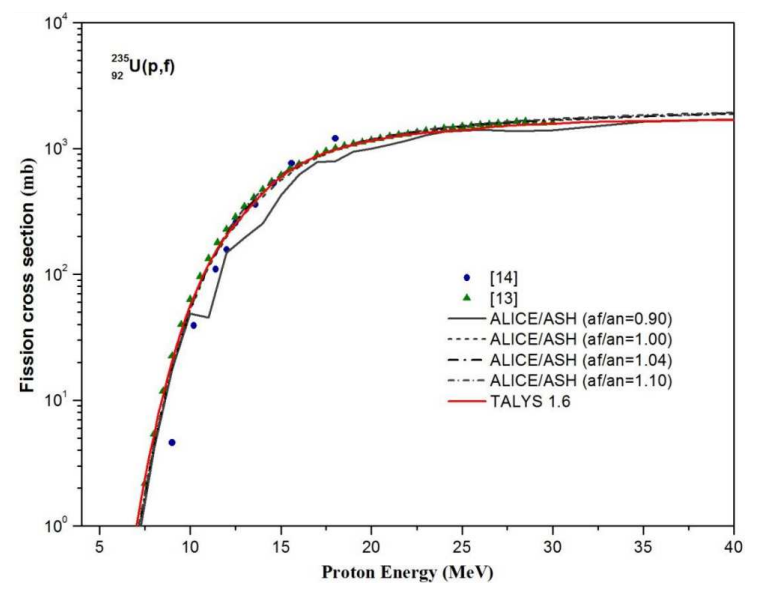

Fig. 3. As in Fig. 1, for ${ }^{235} \mathrm{U}$.

\section{Simulation software}

ALICE/ASH code is a modified version of the ALICE code, with additional pyhsics, capabilities and corrections. The fission cross section computed with ALICE/ASH-2006 is dependent on the choice of $a_{f} / a_{n}$,

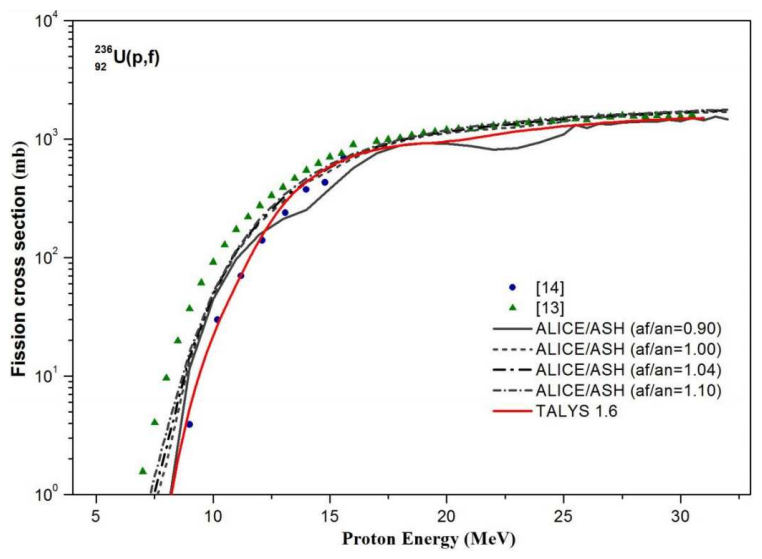

Fig. 4. As in Fig. 1, for ${ }^{236} \mathrm{U}$.

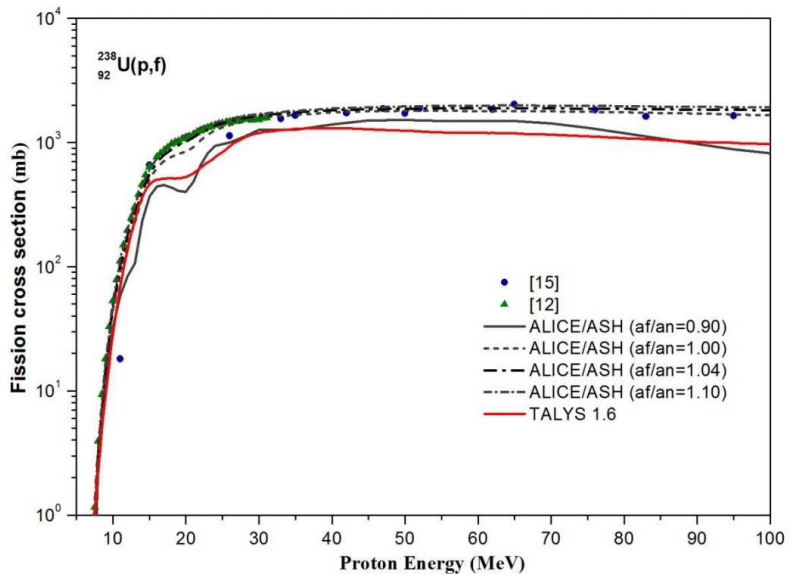

Fig. 5. As in Fig. 1, for ${ }^{238} \mathrm{U}$.

which is the ratio of saddle point to after neutron evaporation level densities. Because of the deformation at the saddle point, this ratio is expected to be larger than 1.0. The use of this ratio accounts, in an effective way, for a change in the intrinsic level density parameter as well as in the rotational enhancement.

Another code, TALYS is a nuclear reaction simulation computer code system for the analysis and prediction of nuclear reactions. The basic objective behind its construction is the simulation of nuclear reactions that involve neutrons, photons, protons, deuterons, tritons, ${ }^{3} \mathrm{He}$-and alpha particles. TALYS integrates the optical model, direct, pre-equilibrium, fission and statistical nuclear reaction models in one calculation scheme and gives a prediction for all open reaction channels.

\section{Results and discussions}

The calculated proton-induced fission cross sections for uranium isotopes at different level density parameter ratios by using both ALICE/ASH code and TALYS 1.6 code are shown in Figs.1-5. The ALICE/ASH and TALYS 1.6 code calculation results were compared with the available experimental data. It can be seen that the 
calculated fission cross sections are found to be remarkably sensitive to the variation of the $a_{f} / a_{n}$. Proton induced fission cross sections increase sharply with the increasing incident photon energy up to a certain value and then continue to increase slowly.

\section{Conclusions}

The calculated fission cross sections are found to be remarkably sensitive to the variation of the $a_{f} / a_{n}$. Protoninduced fission cross sections increase sharply with increasing incident proton energy up to a certain value and then continue to increase slowly. It seems one can not exactly describe the fission cross sections with a single value of level density parameter ratio $\left(a_{f} / a_{n}\right)$. A few percent variations from the optimal value of the level density parameter ratio change the value of the cross section to a large extent. To get a good description of the measured fission cross sections for uranium isotopes, we need to use a ratio of the level density parameters in the fission, $a_{f} / a_{n}$ depending on both, the target nucleus and on the energy of the projectile.

\section{References}

[1] P. Möller, A.J. Sierk, A. Iwamoto, Phys. Rev. Lett. 92, 072501 (2004).

[2] A.J. Koning, S. Hilaire, S. Goriely, Nucl. Phys. A 810, 13 (2008).

[3] O.A.P. Tavares, E.L. Medeiros, J. Phys. G: Nucl. Part. Phys. 30, 395 (2004).

[4] N. Karpuz, M.C. Boz, B. Mavi, F. Öner, İ. Akkurt, Acta Phys. Pol. A 128, B-363 (2015).
[5] N. Karpuz, İ. Akkurt, B. Mavi, Ann. Nucl. En. 60, 341 (2013).

[6] İ. Akkurt, N. Karpuz, B. Mavi, N. Demir, Ann. Nucl. En. 65, 181 (2014).

[7] N. Karpuz, M.C. Boz, B. Mavi, İ. Akkurt, Acta Phys. Pol. A, 128, B-409 (2015).

[8] N. Karpuz, B. Mavi, İ. Akkurt, Acta Phys. Pol. A 128, B-414 (2015).

[9] C.H.M. Broeders, A.Yu. Konobeyev, Yu.A. Korovin, V.P. Lunev, M. Blann, ALICE/ASH - PreCompound and evaporation model code system for calculation of excitation functions, energy and angular distributions of emitted particles in nuclear reactions at intermediate energies, User Manual, 2006.

[10] A. Koning, S. Hilaire, S. Goriely, TALYS 1.6 - A nuclear reaction program, User Manual, 2013.

[11] EXFOR/CSISRS, Experimental Nuclear Reaction Data File, Brookhaven National Laboratory, National Nuclear Data Center, 2009.

[12] J.R. Boyce, Ph.D. Thesis, University Microfilms Order, 1972.

[13] J.R. Boyce, T.D. Hayward, R. Bass, H.W. Newson, E.G. Bilpuch, F.O. Purser, H.W. Schmitt, Phys. Rev. C, Nucl. Phys. 10, 231, (1974).

[14] T. Ohtsuki, Y. Nagame, K. Tsukada, N. Shinohara, S. Baba, K. Hashimoto, I. Nishinaka, K. Sueki, Y. Hatsukawa, K. Hata, T. Sekine, I. Kanno, H. Ikezoe, H. Nakahara, Phys. Rev. C, Nucl. Phys. 44, 1405 (1991).

[15] A.N. Smirnov, I.Yu. Gorshkov, A.V. Prokofiev, V.P. Eismont, in: Conf: 21. Int. Symp. on Nucl. Phys., Gaussig 1991, p. 214. 